\title{
The Effect of Inconsistent Product Attribute Reviews on Consumers' Purchase Intention
}

\author{
Ding Pan, Ya Xu, Yanyan Wu \\ School of Management, Jinan University, Guangzhou, China \\ Email: xuyaadam@126.com
}

How to cite this paper: Pan, D., Xu, Y., \& Wu, Y. Y. (2017). The Effect of Inconsistent Product Attribute Reviews on Consumers' Purchase Intention. Psychology, 8, 2187-2199. https://doi.org/10.4236/psych.2017.813139

Received: October 26, 2017

Accepted: November 26, 2017

Published: November 29, 2017

Copyright $\odot 2017$ by authors and Scientific Research Publishing Inc. This work is licensed under the Creative Commons Attribution International License (CC BY 4.0).

http://creativecommons.org/licenses/by/4.0/

\begin{abstract}
This paper studies how the inconsistent product attributes reviews affect the consumer purchase intention, and discusses the mediating role of information processing fluency and the moderating role of the relationship norm and the ambiguity tolerance. In this study, 3 experiments were conducted to examine the significant effect of inconsistent product attribute reviews on the purchase intention, and the mediating effect of information processing fluency. The relationship between the relationship norm and ambiguity tolerance was also examined. The experimental results validate the research hypothesis, and the research also discusses the theoretical contribution, the management inspiration and the future research direction.
\end{abstract}

\section{Keywords}

Ambivalence Online Review, Purchase Intention

\section{Introduction}

Online review is the product evaluation put forward by consumers through the shopping website or the third party review website, which includes the evaluation of product quality, service, logistics, packaging, and even the purchase and use of a particular product or service by a consumer. The content of online reviews covers a wide range of products, such as product quality, price, service attitude, environmental atmosphere, appearance style and so on, providing a lot of product information and usage information for other consumers' reference decision. The current study considers online reviews only as positive or negative, without considering positive and negative coexistence. Even if a small number of scholars pay attention to the situation of positive and negative coexistence, there is no further research on the inconsistent content. However, in the real situation, consumers are often faced with positive and negative coexistence, which will 
greatly increase the usefulness of online reviews, and have a great impact on consumers' purchase intention. When faced with the inconsistent reviews, how should the store guide consumers to make purchasing decisions, to enlarge the impact of positive evaluation or correct the image of negative evaluation? Or both? In the face of different characteristics of consumers, how should enterprises communicate with them?

\section{Theoretical Background}

\subsection{Ambivalence Online Review and Purchase Intention}

Simple positive and negative reviews do not constitute inconsistencies in online reviews, but the proportion of positive and negative reviews is the key to constitute inconsistencies (Huang, Feng, \& Xie, 2010). In the positive and negative reviews, the dominant reviews will be affected by the conflict reaction, and the accumulation of conflict will reduce the effectiveness of the leading reviews, finally the contradiction of attitude will rise (Chen \& Chen, 2009). If a product is clearly dominated by negative reviews, consumers will immediately abandon the product and then find better products and services.

Consumers have both positive and negative cognition or emotion about the same attribute of the product, as well as the different attributes of the product. For online reviews, it consists of intra-attribute ambivalence and inter-attribute ambivalence. Intra-attribute ambivalence means "there are positive and negative evaluations toward the same product attribute in inconsistent online reviews of product attribute". Inter-attribute ambivalence means "there are positive and negative evaluations toward the different product attributes in inconsistent online reviews of product attribute".

The cognitive dissonance theory holds that when consumers find inconsistent information, they feel psychological conflict and nervousness, hoping to change the inconsistency through attitude or behavior (Festinger, 1957). When faced with intra-attribute ambivalence, consumers receive both positive and negative reviews of the same product attribute. This direct conflict leads to a greater degree of cognitive dissonance. Consumers do not know how to judge the quality of products and they are more likely to feel the difficulty of information processing (MacDonald \& Zanna, 1998). It could be forecasted that consumers need to spend more time and effort on information processing to make purchase decisions (Lee \& Labroo, 2004). When faced with inter-attribute ambivalence, consumers receive positive and negative reviews of different product attributes. They will produce inconsistent judgement toward different product attributes. This inconsistent judgement results in a low sense of conflict, and thus a low sense of cognitive dissonance. Based on the above analysis, this study proposes the hypothesis 1 :

$\mathrm{H} 1$ : compared to inter-attribute ambivalence, the intra-attribute ambivalence has a more significant impact on consumers' purchase intention, more able to reduce consumers' purchase intention. 


\subsection{The Mediating Effect of Information Processing Fluency}

The information processing fluency refers to the difficulty degree of subjective perception in the process of handling object information (Novemsky et al., 2007). The key concept fluency of this study refers to the difficulty of identifying target stimuli through semantic analysis (Hamann, 1990; Whittlesea, 1993). There is a positive evaluation of products, but also negative evaluation of products in inconsistent online reviews of product attribute. The process of reading online reviews is the process of parsing semantics and consumers differ in the degree of difficulty in semantic parsing. That is, inconsistent product attribute reviews will affect the consumer's information processing fluency.

When consumers are confronted with intra-attribute ambivalence, their product knowledge is not enough to make them satisfied with their decisions, which allows consumers to perceive greater risk. In order to mitigate this risk perception, consumers will search for more information to avoid psychological burdens, and try to avoid information that may cause cognitive dissonance in order to improve the satisfaction of purchasing decisions. That is, faced with intra-attribute ambivalence, consumers will produce greater psychological conflict and their information processing fluency is also low. As to make a satisfactory purchase decision, they will spend more time and energy into the semantic parsing and information searching. When faced with inter-attribute ambivalence, the inconsistency is small, and consumers will produce a small psychological conflict. The information processing fluency is relatively high, and they will spend less time and energy into the semantic parsing and information searching to make a purchase decision. Studies have shown that people are more likely to produce buying behavior when they are fluent in external information. When people feel higher information processing fluency, they think their behavior is "right", which leads to their subsequent behavior enhancement (Avnet, 2006). When people feel lower information processing fluency, they will spend more time gathering and analyzing information, easier to postpone decision making (Novemsky et al., 2007).

Through the above analysis, it is found that different types of inconsistent product attribute reviews will lead to different degrees of information processing fluency and different degree of influence on consumers' purchase intention. Based on the above analysis, this study puts forward hypothesis 2 :

$\mathrm{H} 2$ : The information processing fluency plays a mediating role in the effect of inconsistent product attribute reviews on consumers' purchase intention.

\subsection{The Moderating Effect of Relationship Norm}

The relationship norm refers to the expectation of the consumer and the enterprise on the behavior involving the relationship between them (Aggarwal, 2004). Mara, Edwards, Clark and Mills (1993) put forward two kinds of relationship norms-transaction relationship norm and common relationship norm. The core difference between the two relationship norms is: when consumers and enter- 
prises establish the transaction relationship norm, consumers will be based on the value of the exchange of expectations and perceptions to take the corresponding behavior, that is, the individual hope that the benefits paid can be a corresponding fair return. This relationship is similar to a businessman's partnership. When consumers and enterprises establish a common relationship norm, consumers' pay is to maintain the relationship and meet others' needs. Consumers are not only concerned about their own interests, but also will take the initiative to understand the needs of enterprises, and this relationship is similar to friends, family communication patterns.

Specifically, when consumers and enterprises are in the transaction relationship norm, consumers will compare their input with output. When the input is greater than output, the consumer will be less willing to buy, but when the output is greater than input, consumers will be more willing to buy (Aggarwal, 2004). The intra-attribute ambivalence may increase the consumer's resistance and skepticism (Kirmani \& Zhu, 2007), and the information processing fluency becomes low. Consumers may suffer a big loss with high perceived risk. The purchase cost may be higher than the purchase benefit. In this case, consumers believe that the input is greater than output, and consumers are less willing to buy. Conversely, when faced with inter-attribute ambivalence, the information processing fluency becomes high. Consumers may suffer a small loss with low perceived risk. On the other hand, the relationship between the consumer and the enterprise or the brand is similar to friendship. Consumers have strong emotional connection and high trust (Grégoire \& Fisher, 2008), and want to maintain their friendly relationship (Stutman \& Newell, 1990). At the same time, in the common relationship norm, consumers will stand in the corporate perspective. They hope that enterprises can achieve better results, and they are willing to make it. According to the theory of benign conflict, benign evaluation refers to the subjective perception of normative, acceptable and reasonable things. When faced with intra-attribute ambivalence, the same attribute has both positive evaluation and negative evaluation. In the case of common relationship norm, consumers have a stronger sense of trust to the enterprise. They have a higher self-explanatory mechanism and basis. They will also produce a benign evaluation when they feel the conflict, thus considering the conflict reasonable and acceptable. When faced with inter-attribute ambivalence, different attributes have both positive evaluation and negative evaluation. Consumers lack the basis of benign explanations when they explain themselves. The information processing fluency is lower, and the perceived risk is higher, therefore the purchase intention will be weaker.

It can be seen that different relationship norms will moderate the effect of inconsistent product attribute reviews on the information processing fluency, which leads to different influence of consumers' purchase intention. Based on the above analysis, this study puts forward hypotheses:

H3a: In the case of transaction relationship norm, the intra-attribute ambivalence can reduce the information processing fluency and thus reduce consumers' 
purchase intention.

H3b: In the case of common relationship norm, the inter-attribute ambivalence can reduce the information processing fluency and thus reduce the consumers' purchase intention.

\subsection{The Moderating Effect of Ambiguity Tolerance}

Ambiguity tolerance is often regarded as a personality trait in which individuals tend to perceive and respond to vague situations or stimuli (Zenasni \& Lubart, 2008). It refers to the way in which individuals perceive and process the vague impressions caused by inconsistent clues when confronted with inconsistent clues (Furnham \& Ribchester, 1995). McLain argues that "vagueness" means that consumers do not understand the inconsistent clues, while "tolerance" means "grudging acceptance" and "intolerance" means "refusal". When faced with inconsistentt reviews, consumers need to consider the complex situations of many positive and negative clues simultaneously, and the situation presents inconsistent elements to form difficult situations. Finally they are in a "ambiguity" state.

When the consumers' ambiguity tolerance is high, consumers will think of the inconsistent product attribute reviews as appealing, challenging and interesting. They will devote more time and energy to information processing, while more likely to obtain a high level of information processing fluency, thus they have a strong desire to buy. When faced with inter-attribute ambivalence, according to the theory of benign conflict, consumers will consider this kind of inconsistency as a benign evaluation, and the conflict will be considered reasonable and acceptable. However, when faced with intra-attribute ambivalence, the benign evaluation is not established. This is because the inconsistent product attribute reviews indirectly indicates that the product has a certain attribute that is controversial, in which case the consumer perceives a greater risk and the willingness to buy is reduced. When the consumer's ambiguity tolerance is low, consumers will perceive inconsistent product attribute reviews as threatening and unacceptable, consumers whose ambiguity tolerance is low will tend to give up to circumvent the potential risks, and will not spend more time and effort clarifying the reasons for this inconsistency. So in this case, whether it is intraattribute ambivalence or inter-attribute ambivalence, consumers will perceive a low level of information processing fluency, and they have a low purchase intention.

Thus, the ambiguity tolerance will moderate the relationship between the inconsistent product attribute reviews and information processing fluency and then affect consumers purchase intention. Based on the above analysis, the following hypotheses are proposed:

H4a: When the consumer' ambiguity tolerance is high, the intra-attribute ambivalence can reduce the information processing fluency and thus reduce consumers' purchase intention.

H4b: When consumers' ambiguity tolerance is low, there is no difference in the effect of inconsistent product attribute reviews on the information processing 
fluency.

\section{Experiment Design}

Based on the above analysis, this study will verify the hypothesis by three experiments. Experiment 1 explores the effects of inconsistent product attribute reviews on consumers' purchase intention and the mediating effect of information processing fluency $(\mathrm{H} 1$ and $\mathrm{H} 2)$ from the perspective of information processing. In experiment 2, it is discussed whether the effects will be changed ( $\mathrm{H} 3 \mathrm{a}$ and $\mathrm{H} 3 \mathrm{~b}$ ) after adding the relationship norm, and the mediating effect of information processing fluency can be further validated. Experiment 3 mainly examines whether the different characteristics of consumers will influence the main effect and the mediating effect ( $\mathrm{H} 4 \mathrm{a}$ and $\mathrm{H} 4 \mathrm{~b})$.

\subsection{Experiment 1}

The purpose of experiment one is to verify the effect of inconsistent product attribute reviews on consumers' purchase intention ( $\mathrm{H} 1)$, and to test the mediating effect of information processing fluency $(\mathrm{H} 2)$. In order to determine the suitable control material, this research has carried out the preliminary experiment. To avoid the confounding effects of real brands, we use virtual brands in our labs. We use the handset because the handset is consumers' very familiar product category, and it is also relatively easy to cause inconsistent product attribute reviews. Through the pre-test one, we established the cell phone's representative product attributes as stimulus, respectively configuration performance and exterior design. Through the pre-test two, we determined that the stimulation of inconsistent product attribute reviews was successful.

Experiment one adopts single factor (inconsistent product attribute reviews: inter-attribute ambivalence VS intra-attribute ambivalence) inter-group design. and the dependent variable is the purchase intention. The experiment uses the scene simulation method to study the consumer's buying behavior in the specific situation. Then, the trial is required to fill in the operating test scale about the inconsistent product attribute reviews (Jeyakumar et al., 1999), information processing fluency (Lee \& Larbroo, 2004), and purchase intention (Sweeney et al., 1999). The participants were asked to fill in demographic information, including gender, age, occupation, and familiarity with the mobile phone and the importance attached to the product attributes in reviews.

\subsection{Results}

Through SPSS data analysis, we find that the formal scale has good reliability and validity. Because, in the inter-attribute ambivalence, scores of inter-attribute ambivalence are higher than those of intra-attribute ambivalence, $P=0.001$. In the intra-attribute ambivalence, scores of intra-attribute ambivalence are higher than those of inter-attribute ambivalence, $P=0.001$. So we know that the manipulation of inconsistent product attribute comment is successful. Experimental 
one is based on single factor (inconsistent product attribute reviews: inter-attribute ambivalence vs. intra-attribute ambivalence) inter-group design.

The manipulation of inter-attribute ambivalence is marked as 0 , and the manipulation of intra-attribute ambivalence is marked as 1 . We use the inconsistent product attribute reviews as the independent variable, and purchase intention is the dependent variable, and then we do the variance analysis. The analysis results show that the main effect is significant, $\mathrm{F}(1,62)=135.023, P=0.000<0.05$, supporting H1. Compared to inter-attribute ambivalence, the purchase intention will be weaker after reading the intra-attribute ambivalence. As shown in Figure 1 .

The mean value of 4 items of information processing fluency is taken as the value of the information processing fluency. The Bootstrap method based on Hayes (2013) is used to test the mediating effect of information processing fluency. First, the sample size is set to 5000, and under the confidence interval of $95 \%$, the results of the mediation test do not include 0 (LLCT $=-1.3067$, ULCI $=$ -0.3248 ), which indicates that the mediating effect of information processing fluency is significant.

At the same time, in order to further test the mediating effect of information processing fluency, we use Baron and Kenny (1986) method to carry on the regression analysis. With the purchase intention as the dependent variable, the inconsistent product attribute reviews as the independent variable, the regression result shows that the adjusted $\mathrm{R}$ is 0.176 , and the model is significant, $P=0.000$. The regression is significant. The coefficient is $-1.648, P=0.000$. With the information processing fluency as the dependent variable, the inconsistent product attribute reviews as the independent variable, the regression result shows that the adjusted $\mathrm{R}$ is 0.192 and the model is significant, $P=0.000$. The regression is significant. The coefficient is $-0.883, P=0.002$. With the purchase intention as the dependent variable, the inconsistent product attribute reviews and the information processing fluency as the independent variables, the regression result shows that the adjusted $\mathrm{R}$ is 0.395 , and the model is significant, $P=0.000$. The regression is significant. The coefficient is $-0.916, P=0.012$, but the significance reduced. The specific regression results are as shown in Table 1.

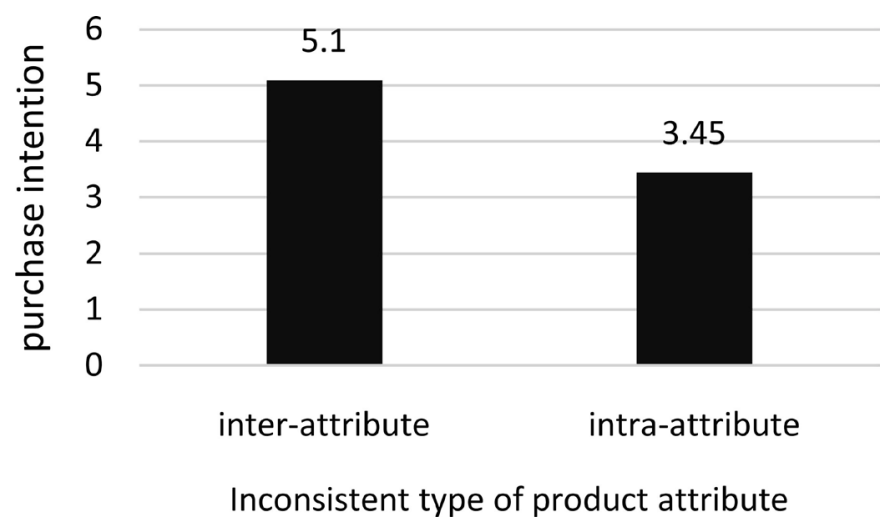

Figure 1. Effect of inconsistent product attribute reviews on purchase intention. 
Table 1. Inconsistent product attribute reviews * information processing fluency.

\begin{tabular}{|c|c|c|c|c|c|c|}
\hline \multirow{3}{*}{ variable } & \multicolumn{2}{|c|}{ Model 1} & \multicolumn{2}{|c|}{ Model 2} & \multicolumn{2}{|c|}{ Model 3} \\
\hline & \multicolumn{2}{|c|}{ Purchase intention } & \multicolumn{2}{|c|}{$\begin{array}{c}\text { Information processing } \\
\text { fluency }\end{array}$} & \multicolumn{2}{|c|}{ Purchase intention } \\
\hline & $\begin{array}{c}\text { Coefficient } \\
\beta\end{array}$ & $\begin{array}{c}\text { Significance } \\
\quad P\end{array}$ & $\begin{array}{c}\text { Coefficient } \\
\beta\end{array}$ & $\begin{array}{c}\text { Significance } \\
\quad p\end{array}$ & $\begin{array}{c}\text { Coefficient } \\
\beta\end{array}$ & $\begin{array}{c}\text { Significance } \\
P\end{array}$ \\
\hline $\begin{array}{l}\text { Inconsistent product } \\
\text { attribute reviews }\end{array}$ & -1.648 & 0.000 & -0.883 & 0.000 & -0.916 & 0.000 \\
\hline $\begin{array}{c}\text { Information } \\
\text { processing fluency }\end{array}$ & & & & & 0.385 & 0.001 \\
\hline Adjusted R & \multicolumn{2}{|c|}{0.176} & \multicolumn{2}{|c|}{0.192} & \multicolumn{2}{|c|}{0.395} \\
\hline significance & \multicolumn{2}{|c|}{0.000} & \multicolumn{2}{|c|}{0.002} & \multicolumn{2}{|c|}{0.012} \\
\hline
\end{tabular}

\subsection{Experiment 2}

In experiment 2, we will study the moderating effect of relationship norm on the purchase intention (H3a and h3b), and further verify the main effect and mediating effect. In order to further verify the main effect, experiment 2 will adopt a different experimental material. According to Tu et al. (2007) research on the product type, mobile phones and sports shoes are different types of stimuli representative, so the Experiment 2 will use sports shoes as stimulus. For the start of relationship norm, we use the method of Aggarwal (2004) and Huang et al. (2009) to manipulate the scene simulation method. Similar to experiment one, we conduct a preliminary experiment. Through the pre-test one, we established the sports shoes' representative product attributes as stimulus, respectively fashion and wear resistance. Through the pre-test two, we determined that the stimulation of inconsistent product attribute reviews was successful.

Experiment 2 adopts the inter-group factor design of 2 (inconsistent product attribute reviews: inter-attribute ambivalence vs. intra-attribute ambivalence) $* 2$ (relationship norm: transaction relationship norm vs. common relationship norm). This experiment uses the situational hypothesis to inspire the relationship norm between the trial and the shop (Aggarwal, 2004; Huang et al., 2009). The existing research shows that the experimental method can initiate the corresponding relationship norm (Clark, 1986). Then, it is similar to experiment one, but we increase the operating test scale of the relationship norm (Aggarwal, 2004).

\subsection{Results}

Through SPSS data analysis, the formal scale has good reliability and validity, and the manipulation of inconsistent product attribute reviews and the relationship norm is also successful.

The analysis results show that the inconsistent product attribute comment has significant effect on purchase intention, $\mathrm{F}(1,99)=144.3, P=0.034<0.05$, supporting H1. With reference to the Bootstrap method proposed by Hayes (2013), the results of the mediating test do not include 0 (LLCT $=-0.424$, ULCI $=$ 
$-0.053)$, indicating that the mediating effect is significant. Like experiment 1 , we use Baron and Kenny (1986) method to carry on the regression analysis and the results support $\mathrm{H} 2$.

The common relationship norm is marked as 0 , and the transaction relationship norm is marked as 1 . First, we examine the moderating effect of relationship norm, the results show that the interaction of inconsistent product attribute reviews * relationship norm is significant, $\mathrm{F}(1,99)=18.949, P=0.000$. As shown in Figure 2, in the case of transaction relationship norm, the information processing fluency of inter-attribute ambivalence is higher, $P=0.007$, supporting $\mathrm{H} 3 \mathrm{a}$, and in the case of common relationship norm, the information processing fluency of intra-attribute ambivalence is higher, $P=0.001$, supporting $\mathrm{H} 3 \mathrm{~b}$.

\subsection{Experiment 3}

The purpose of experiment three is to examine whether the consumers' different ambiguity tolerance will influence purchase intention when faced with inconsistent product attribute reviews ( $\mathrm{H} 4 \mathrm{a}$ and $\mathrm{H} 4 \mathrm{~b}$ ). In order to guarantee the reliability and validity of the experimental materials, the stimuli are similar to experiment 2. Experiment 3 adopts single factor (inconsistent product attribute reviews: inter-attribute ambivalence VS intra-attribute ambivalence) inter-group design. Then, it is similar to experiment one, but we increase the operating test scale of ambiguity tolerance measurement scale (Stanley Budner, 1962).

\subsection{Results}

According to SPSS data analysis, the formal scale has good reliability and validity, and the manipulation of inconsistent product attribute reviews is successful. The main effect and mediating effect also proved remarkable. Then the regulation of ambiguity tolerance is proved. The mean value of the 16 questions of the

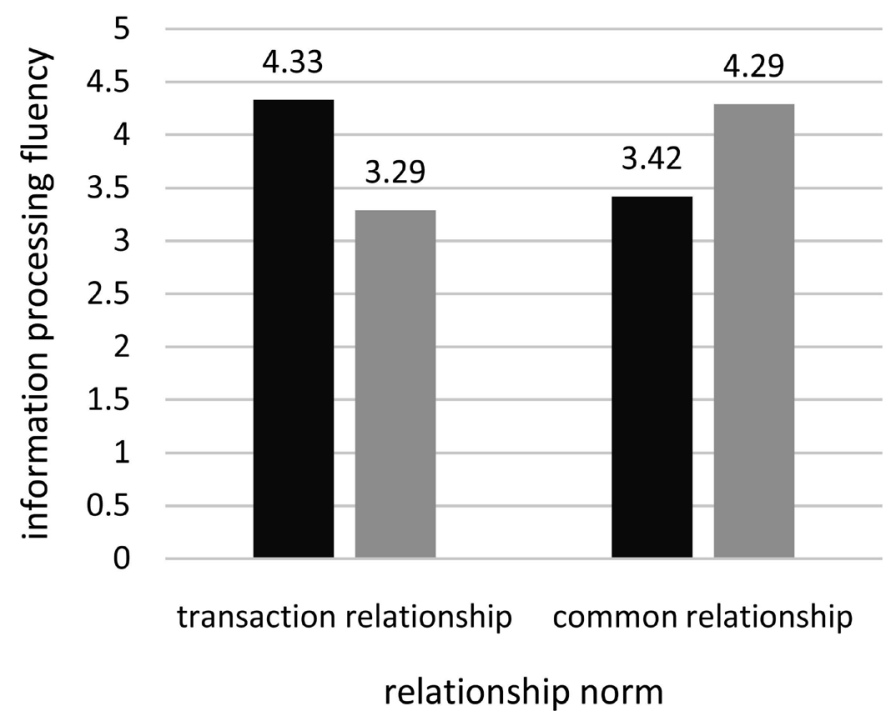

inter-attribute intra-attribute

Figure 2. The moderating effect of relationship norm. 
6

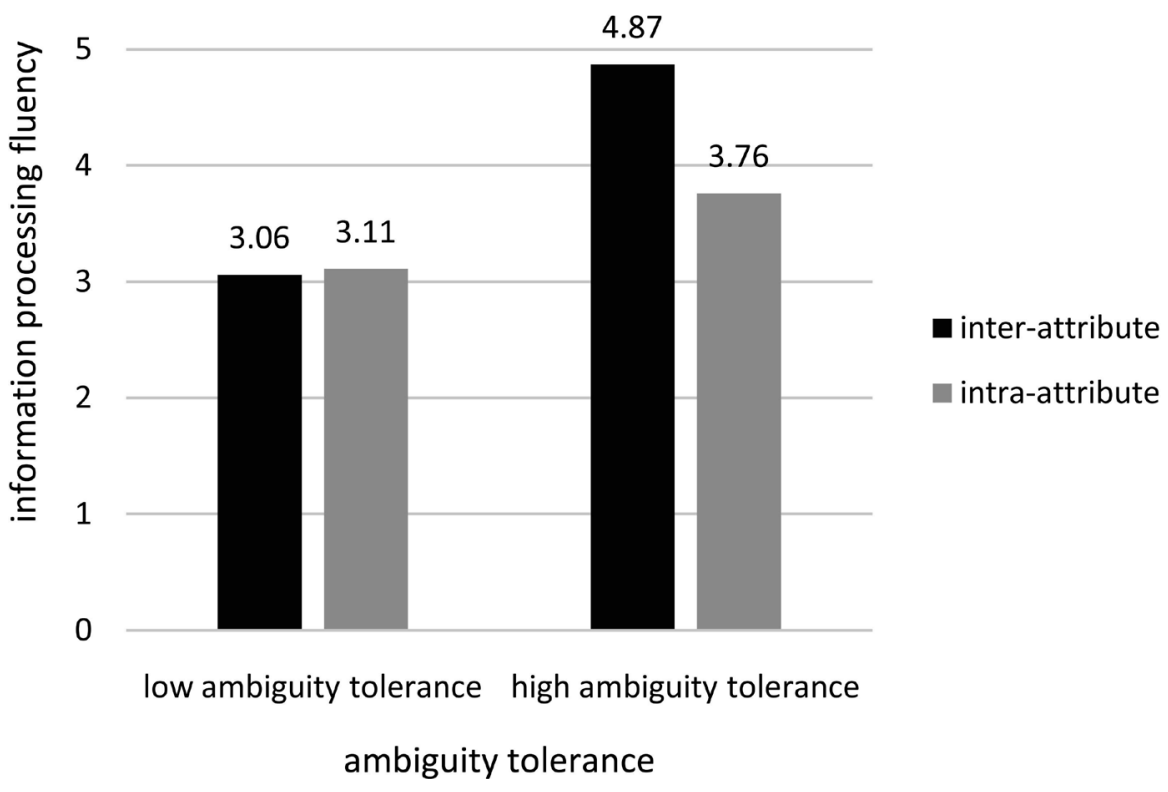

Figure 3. The moderating effect of ambiguity tolerance.

ambiguity tolerance is taken as the value of the ambiguity tolerance degree. First, we examine the moderating effect of ambiguity tolerance, the results show that the interaction of inconsistent product attribute reviews * the ambiguity tolerance is significant, $\mathrm{F}(1,97)=19.442, P=0.000$.

Details as shown in Figure 3, when consumers' ambiguity tolerance is low, there is no difference in the information processing fluency of inter-attribute ambivalence and intra-attribute ambivalence, $P=0.818>0.05$, supporting H4a, and when consumers' ambiguity tolerance is higher, the information processing fluency of inter-attribute ambivalence is higher, $P=0.000$, supporting H4b.

\section{General Discussion}

From the source of inconsistent reviews, Experiment one shows that the main effect is significant, $\mathrm{F}(1,62)=135.023, P=0.000<0.05$, supporting that inconsistent product attribute reviews have a significant impact on purchase intention. Compared to inter-attribute ambivalence, intra-attribute ambivalence has a greater influence on information processing fluency, and the purchase intention is lower. Through research we can find that in the case of transaction relationship norm, the information processing fluency of inter-attribute ambivalence is higher, $P=0.007$, the intra-attribute ambivalence can reduce the information processing fluency and thus reduce consumers' purchase intention. In the case of common relationship norm, the information processing fluency of intraattribute ambivalence is higher, $P=0.001$, the inter-attribute ambivalence can reduce the information processing fluency and thus reduce the consumers' purchase intention. When the consumer's ambiguity tolerance is high, the information processing fluency of inter-attribute ambivalence is higher, $P=0.000$. The 
intra-attribute ambivalence can reduce the information processing fluency and reduce the consumers' purchase intention. When consumers' ambiguity tolerance is low, there is no difference in the information processing fluency of interattribute ambivalence and intra-attribute ambivalence, $P=0.818>0.05$. The inconsistent product attribute reviews have no significant effect on the information processing fluency.

This study has the following implications for future research: from the information processing angle. It enriches the research of inconsistent product attribute reviews. Considering the individual characteristics of consumers and the relationship between consumers and enterprises, this paper provides a new theoretical perspective for future research.

\section{Managerial Implications and Limitations}

From the problems about inconsistent reviews that consumers encounter in online shopping, the study could solve the problem through model construction and empirical research, which has an important enlightenment to the enterprise management practice.

The merchants had better prioritize the intra-attribute ambivalence. According to the study, the intra-attribute ambivalence is more likely to reduce the buyer's purchase intention than the inter-attribute ambivalence. Therefore, when dealing with inconsistent online reviews, the merchants should prioritize the intra-attribute ambivalence and reduce the negative impact that may occur.

The relationship norm adjusts the effect of inconsistent product attribute reviews on consumers' purchase intention. As the connection between enterprises and consumers, the relationship norm plays an important role in the interaction between enterprises and consumers. The common relationship norm essentially alleviates consumers' sense of conflict in the face of inconsistent product attribute reviews, and enhances the consumers' self-explanation and trust to the enterprise. While in the case of transaction relationship norm, the consumer and the enterprise pay for the corresponding return. Therefore, they do not have the sense of trust. When faced with the inconsistent reviews, they still perceive a high sense of conflict. Therefore, the good relationship between enterprises and consumers helps to improve the efficiency of interaction with consumers.

Faced with consumers of different ambiguity tolerance, the merchants should promptly respond to consumers' online consultation and communicate actively with consumers. They should understand the causes of consumer ambivalence, and take different measures for different consumers. For consumers with high ambiguity tolerance, they should be patient with possible confusion and proactively answer information that consumers may face. They have to minimize the psychological conflict of consumers, and improve their information processing fluency. For consumers with low ambiguity tolerance, it may not be enough to save the hearts of consumers simply from the perspective of product information. The merchants can try to win this group in terms of product benefits and 
product discounts.

Negative reviews are more persuasive to potential consumers than positive online reviews, and we should avoid negative reviews on the following aspects: 1) Pre-purchase precautions. To fully inform the consumer of the function of the product and the emotional pleasure that may be acquired before purchasing, and avoid fraud; 2) Negative reviews should be dealt with in a timely manner, with particular emphasis on the review of remedies, whether it is inter-ambivalence or intra-ambivalence. When there are negative comments, you should respond in time to improve the customer's degree of goodwill.

As far as this research is concerned, limited to time, method and data, there are still many deficiencies in this research, which need to be further expanded and deepened in future research. The sample size and the research object need to be enlarged. Although this research sample satisfies the research needs, the increase of sample size will be able to achieve more ideal results. In addition, the object of this study is the university students, although they are the representative power of the network shopping, and it is better to expand the sample object, including the white-collar workers, the worker and so on. The experimental conditions are limited. Due to the limitations of experimental conditions, we do not have a real shopping scene. We use the questionnaires to investigate in the laboratory simulating a shopping scene. If the conditions allow, consumers' shopping choice under the real shopping scene will be able to better explain the relationship between the inconsistent product attribute reviews and the purchase intention. The elimination of control variables needs to be more rigorous. This study tries to avoid the effect of price, brand, rating and other factors on consumers' purchase intention. However, the influence of these factors on consumers' purchase intention is still not completely excluded, and the research conclusions will be affected to some extent.

\section{References}

Aggarwal, P. (2004). The Effects of Brand Relationship Norms on Consumer Attitudes and Behavior. Journal of Consumer Research, 31, 87-101. https://doi.org/10.1086/383426

Avnet, T., \& Higgins, E. T. (2006). How Regulatory Fit Affects Value in Consumer Choices and Opinions. Journal of Marketing Research, 43, 1-10. https://doi.org/10.1509/jmkr.43.1.1

Chen, J., \& Chen, Z. (2009). A Review of the Research on General Ambivalent Attitude. Psychological Science, 32, 220-222.

Clark, D. M. (1986). A Cognitive Approach to Panic. Behaviour Research and Therapy, 24, 461 -470. https://doi.org/10.1016/0005-7967(86)90011-2

Festinger, L. (1957). A Theory of Cognitive Dissonance Evanston. IL: Row, Peterson.

Furnham, A., \& Ribchester, T. (1995). Tolerance of Ambiguity: A Review of the Concept, Its Measurement and Applications. Current Psychology, 14, 179-199. https://doi.org/10.1007/BF02686907

Grégoire, Y., \& Fisher, R. J. (2008). Customer Betrayal and Retaliation: When Your Best Customers Become Your Worst Enemies. Journal of the Academy of Marketing 
Science, 36, $247-261$. https://doi.org/10.1007/s11747-007-0054-0

Hamann, S. B. (1990). Level-of-Processing Effects in Conceptually Driven Implicit Tasks. Journal of Experimental Psychology: Learning, Memory, and Cognition, 16, 970. https://doi.org/10.1037/0278-7393.16.6.970

Huang, M., Cai, F., Zhou, Y., \& Zhu, H. (2009). The Role of Relationship Norms in Consumers' Complaint Intention. Acta Psychologica Sinica, 41, 989-999.

Huang, M., Feng, X., \& Xie, T. (2010). New Understanding of Consumer Attitude: Dualistic Attitudinal Ambivalence. Advances in Psychological Science, 18, 987-996.

Jeyakumar, M., Butters, T. D., Cortina-Borja, M., Hunnam, V., Proia, R. L., Perry, V. H. et al. (1999). Delayed Symptom Onset and Increased Life Expectancy in Sandhoff Disease Mice Treated with N-Butyldeoxynojirimycin. Proceedings of the National Academy of Sciences, 96, 6388-6393.

Kirmani, A., \& Zhu, R. (2007). Vigilant against Manipulation: The Effect of Regulatory Focus on the Use of Persuasion Knowledge. Journal of Marketing Research, 44, 688 -701. https://doi.org/10.1509/jmkr.44.4.688

Lee, A. Y., \& Labroo, A. A. (2004). The Effect of Conceptual and Perceptual Fluency on Brand Evaluation. Journal of Marketing Research, 41, 151-165. https://doi.org/10.1509/jmkr.41.2.151.28665

MacDonald, T. K., \& Zanna, M. P. (1998). Cross-Dimension Ambivalence toward Social Groups: Can Ambivalence Affect Intentions to Hire Feminists? Personality and Social Psychology Bulletin, 24, 427-441. https://doi.org/10.1177/0146167298244009

Mara, D. D., Edwards, P., Clark, D., \& Mills, S. W. (1993). A Rational Approach to the Design of Wastewater-Fed Fishponds. Water Research, 27, 1797-1799. https://doi.org/10.1016/0043-1354(93)90120-7

Novemsky, N., Dhar, R., Schwarz, N. et al. (2007) Preference Fluency in Choice. Journal of Marketing Research, 44, 347-356. https://doi.org/10.1509/jmkr.44.3.347

Stanley Budner, N. Y. (1962). Intolerance of Ambiguity as a Personality Variable. Journal of Personality, 30, 29-50.

Stutman, R. K., \& Newell, S. E. (1990). Rehearsing for Confrontation. Argumentation, 4, 185-198. https://doi.org/10.1007/BF00175422

Sweeney, J. C., Soutar, G. N., \& Johnson, L. W. (1999). The Role of Perceived Risk in the Quality-Value Relationship: A Study in a Retail Environment. Journal of Retailing, 75, 77-105.

Tu, R., Zhao, Z., \& Tu, P. (2007). The Impact of Product Attributes on Customer Satisfaction: An Empirical Study. Journal of Management Sciences, 36-44.

Whittlesea, B. W. A. (1993). Illusions of Familiarity. Journal of Experimental Psychology: Learning, Memory, and Cognition, 19, 1235. https://doi.org/10.1037/0278-7393.19.6.1235

Zenasni, F., Besançon, M., \& Lubart, T. (2008). Creativity and Tolerance of Ambiguity: An Empirical Study. The Journal of Creative Behavior, 42, 61 -73. https://doi.org/10.1002/j.2162-6057.2008.tb01080.x 